Gazi University
Journal of Science
$\mathrm{http} / /$ dergipark.gov.tr/gujs

\title{
Spectrum and the Jost Solution of Discrete Impulsive Klein-Gordon Equation with Hyperbolic Eigenparameter
}

\author{
Turhan KOPRUBASI* \\ Department of Mathematics, Kastamonu University, Turkey \\ Highlights \\ - This paper focuses on discrete impulsive Klein-Gordon equation with hyperbolic eigenparameter. \\ - Several spectral properties of the spectrum and the scattering function are investigated. \\ - An example about the scattering function and the existence of eigenvalues is given.
}

Article Info

Received: 16 Feb 2021

Accepted: 22 Oct 2021

Keywords

Klein-Gordon equations Impulsive condition Hyperbolic parameter Spectral analysis Scattering function

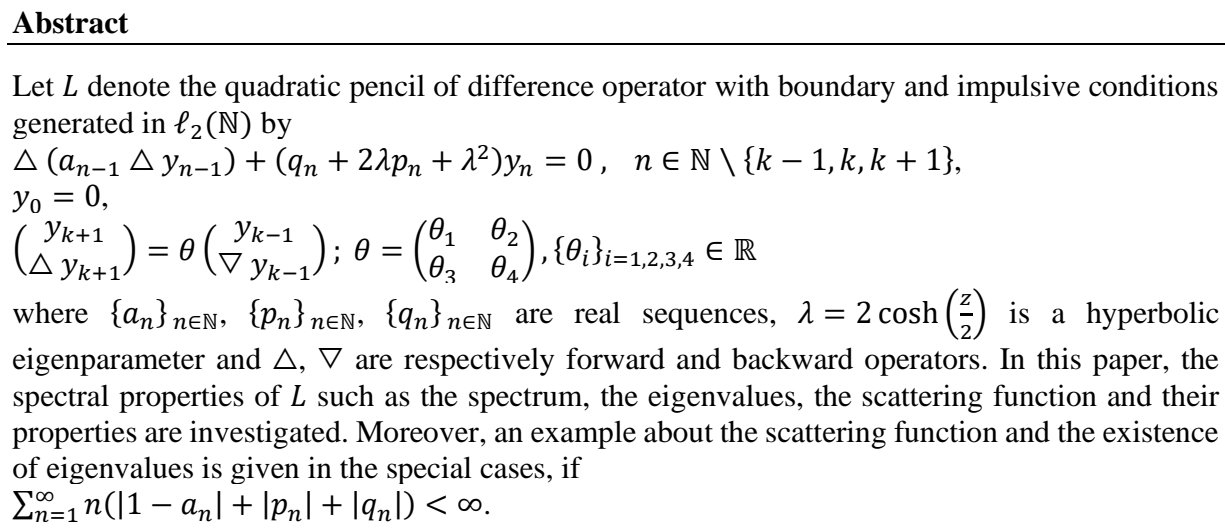

\section{INTRODUCTION}

Spectral theory of the difference equations related with some operators and their inverses is one of the basic branches of modern functional analysis. It is very important in terms of helping to understand many daily events about biology, economics, engineering and physics, especially quantum tunneling and resonances. Because of that, spectrum of the difference equations with spectral singularities have been widely debated by many mathematicians from the recent past to the present ([1-10]).

Let consider the Klein-Gordon problem (KGP)

$$
\begin{aligned}
& y^{\prime \prime}+[\lambda-p(x)]^{2} y=f(x), \quad x \in \mathbb{R}_{+}=[0, \infty] \\
& \alpha y^{\prime}(0)-\beta y(0)=0
\end{aligned}
$$

for a particle zero mass with static complex potential $p$ and complex-valued function $f$ where $\alpha, \beta \in \mathbb{C}$ with $|\alpha|+|\beta| \neq 0$ and $\lambda$ is a spectral parameter. The Jost solution and the spectral properties of the KGP (1) has been investigated in [11] and it is shown that it has finite number of eigenvalues and spectral singularities with finite multiplicities. In additon, the spectral analysis of a quadratic pencil of Schrödinger operator has been investigated in $[12,13]$. In that studies, the authors worked on the spectrum, principal functions correponding to spectral singularities and an eigenfunction expansion of it. Many other studies related to these equation can be seen in [14-19]. Furthermore, the impulsive difference equations, which 
constitute a model for many problems that arise with momentary changes caused by external factors in fields such as pharmacokinetics, ecology, control theory and mechanics, have been the subject of many researchers to understand the nature of such dynamics. The studies of the spectral theory of such equations has become one of the fastest developing branches of mathematics in terms of difference equations ([2025]).

Now, the quadratic pencil difference operator $L$ in $\ell_{2}(\mathbb{N})$ denoted by

$\triangle\left(a_{n-1} \triangle y_{n-1}\right)+\left(q_{n}+2 \lambda p_{n}+\lambda^{2}\right) y_{n}=0, \quad n \in \mathbb{N} \backslash\{k-1, k, k+1\}$,

with boundary and impulsive conditions

$y_{0}=0$,

$\left(\begin{array}{c}y_{k+1} \\ \triangle y_{k+1}\end{array}\right)=\theta\left(\begin{array}{c}y_{k-1} \\ \nabla y_{k-1}\end{array}\right) ; \theta=\left(\begin{array}{cc}\theta_{1} & \theta_{2} \\ \theta_{3} & \theta_{4}\end{array}\right),\left\{\theta_{i}\right\}_{i=1,2,3,4} \in \mathbb{R}$

where $\mathbb{N}$ is set of natural numbers, $\left\{a_{n}\right\}_{n \in \mathbb{N}},\left\{p_{n}\right\}_{n \in \mathbb{N}},\left\{q_{n}\right\}_{n \in \mathbb{N}}$ are real sequences, $a_{n} \neq 0$ for all $n \in \mathbb{N} \cup$ $\{0\}, \operatorname{det} \theta \neq 0, \lambda$ is a hyperbolic eigenparameter and $\triangle, \nabla$ are respectively forward and backward operators. The situation that makes this paper, which is one of the articles have applicability in a lot of branches of bursting rhythm models in medicine and biology, optimal control models in economics, pharmacokinetics and frequency modulated systems, different from other studies in the literature, is the paper of an discrete impulsive Klein-Gordon equation with hyperbolic eigenparameter $2 \cosh \left(\frac{z}{2}\right)$. In this study, various spectral properties of $L$; i.e., the spectrum, the scattering function and their properties are investigated if

$\sum_{n=1}^{\infty} n\left(\left|1-a_{n}\right|+\left|p_{n}\right|+\left|q_{n}\right|\right)<\infty$.

\section{SCATTERING FUNCTION OF $L$}

Let two semi-strips $T_{-}=\{z \in \mathbb{C}: z=\xi+i \tau, \xi<0, \tau \in[0,4 \pi]\}$ and $T=T_{-} \cup T_{0}$ are defined in the closure of $\mathbb{C}_{\text {left }}=\{z \in \mathbb{C}: \operatorname{Re} z<0\}$ where $T_{0}=\{z \in \mathbb{C}: z=i \tau, \tau \in[0,4 \pi]\}$. Clearly, $T=\{z \in \mathbb{C}: z=\xi+i \tau, \xi \leq$ $0, \tau \in[0,4 \pi]\}$. Under the Condition (5), Equation (2) has the solution

$f_{n}(z)=a_{n} e^{n z}\left(1+\sum_{m=1}^{\infty} K_{n m} e^{m^{\frac{z}{2}}}\right), n \in\{k+1, k+2, \ldots\}$,

satisfying the condition $\lim _{n \rightarrow \infty} e^{-n z} f_{n}(z)=1, z \in T_{-}$for $\lambda=2 \cosh \left(\frac{z}{2}\right)$, and $\alpha_{n}, K_{n m}$ are expressed in terms of $\left\{a_{n}\right\}_{n \in \mathbb{N}},\left\{p_{n}\right\}_{n \in \mathbb{N}}$, and $\left\{q_{n}\right\}_{n \in \mathbb{N}}$ as

$$
\begin{aligned}
\alpha_{n}= & {\left[\prod_{\xi=n}^{\infty}\left(-a_{\xi}\right)\right]^{-1} } \\
K_{n, 1}= & 2 \sum_{\xi=n+1}^{\infty} p_{\xi} \\
K_{n, 2}= & \sum_{\xi=n+1}^{\infty}\left(2-a_{\xi}-a_{\xi-1}\right)+2 \sum_{\xi=n+1}^{\infty} p_{\xi} K_{\xi, 1} \\
K_{n, 3}= & \sum_{\xi=n+1}^{\infty}\left(2-a_{\xi}-a_{\xi-1}\right) K_{\xi, 1}+2 \sum_{\xi=n+1}^{\infty} p_{\xi}\left(K_{\xi, 2}+1\right) \\
K_{n, 4}= & \sum_{\xi=n+1}^{\infty}\left[\left(1-a_{\xi}^{2}\right)+\left(2-a_{\xi}-a_{\xi-1}\right) K_{\xi, 2}\right]+2 \sum_{\xi=n+1}^{\infty} p_{\xi}\left(K_{\xi, 1}+K_{\xi, 3}\right) \\
K_{n, m+4}= & K_{n m}+\sum_{\xi=n+1}^{\infty}\left[\left(1-a_{\xi}^{2}\right) K_{\xi+1, m}+\left(2-a_{\xi}-a_{\xi-1}\right) K_{\xi, m+2}\right]+2 \sum_{\xi=n+1}^{\infty} p_{\xi}\left(K_{\xi, m+1}+\right. \\
& \left.K_{\xi, m+3}\right)
\end{aligned}
$$


for $m=1,2, \ldots$ by following up [26]. Moreover

$\left|K_{n m}\right| \leq \delta \sum_{k=n+\llbracket \frac{m}{2} \rrbracket}^{\infty}\left(\left|1-a_{k}\right|+\left|p_{k}\right|+\left|q_{k}\right|\right)$

holds, where $\delta>0$ is constant and $\llbracket \frac{m}{2} \rrbracket$ is the integer part of $\frac{m}{2}$. Hence, $4 \pi i$ periodic function $f_{n}(z)$ is analytic with respect to $z$ in $\mathbb{C}_{\text {left }}$ and continuous on the real axis.

Also, the elementary solutions of $(2)\left\{\varphi_{n}(z)\right\}$ and $\left\{\psi_{n}(z)\right\}, n=0,1, \ldots, k-1$ are considered for $z \in T$ subject to the initial conditions

$\varphi_{0}(z)=0 \quad, \quad \varphi_{1}(z)=1$,

$\psi_{0}(z)=a_{0}^{-1}, \quad \psi_{1}(z)=0$.

These functions are entire for $z \in \mathbb{C}$ and the Wronskian of them can be found as

$$
\begin{aligned}
W\left[\varphi_{n}(z), \psi_{n}(z)\right] & =a_{n}\left[\varphi_{n}(z) \psi_{n+1}(z)-\varphi_{n+1}(z) \psi_{n}(z)\right] \\
& =a_{0}\left[\varphi_{0}(z) \psi_{1}(z)-\varphi_{1}(z) \psi_{0}(z)\right] \\
& =-1
\end{aligned}
$$

In here, the Jost solution of $L$ is defined by using $f_{n}(z), \varphi_{n}(z)$ and $\psi_{n}(z)$

$J_{n}(z)=\left\{\begin{array}{cl}p^{(1)}(z) \varphi_{n}(z)+p^{(2)}(z) \psi_{n}(z) & ; n=0,1, \ldots, k-1 \\ f_{n}(z) & ; n=k+1, k+2, \ldots\end{array}\right.$

for $z \in T$ with

$p^{(1)}(z)=-\frac{a_{k-2}}{\operatorname{det}\left(\theta B^{-1}\right)} \quad, \quad p^{(2)}(z)=\frac{a_{k-2}}{\operatorname{det}\left(\theta C^{-1}\right)}$

where

$$
\begin{aligned}
B & =\left(\begin{array}{cc}
f_{k+1}(z) & \triangle f_{k+1}(z) \\
\theta_{1} \psi_{k-1}(z)+\theta_{2} \nabla \psi_{k-1}(z) & \theta_{3} \psi_{k-1}(z)+\theta_{4} \nabla \psi_{k-1}(z)
\end{array}\right), \\
C & =\left(\begin{array}{cc}
f_{k+1}(z) & \triangle f_{k+1}(z) \\
\theta_{1} \varphi_{k-1}(z)+\theta_{2} \nabla \varphi_{k-1}(z) & \theta_{3} \varphi_{k-1}(z)+\theta_{4} \nabla \varphi_{k-1}(z)
\end{array}\right)
\end{aligned}
$$

obtained from (4). Moreover,

$$
\begin{aligned}
W\left[f_{n}(z), f_{n}(-z)\right] & =\lim _{n \rightarrow \infty}\left\{a_{n}\left[f_{n}(z) f_{n+1}(-z)-f_{n+1}(z) f_{n}(-z)\right]\right\} \\
& =\lim _{n \rightarrow \infty}\left\{a_{n}\left[e^{-z} f_{n}(z) e^{-n z} f_{n+1}(-z) e^{(n+1) z}-e^{z} f_{n+1}(z) e^{-(n+1) z} f_{n}(-z) e^{n z}\right]\right\} \\
& =e^{-z}-e^{z} \\
& =-2 \sinh z
\end{aligned}
$$

for $z \in T_{0} \backslash\{k \pi i: k=0,1,2,3,4\}$. If

$$
F_{n}(z)=\left\{\begin{array}{cl}
\varphi_{n}(z) & ; n=0,1, \ldots, k-1 \\
q^{(1)}(z) f_{n}(z)+q^{(2)}(z) f_{n}(-z) & ; n=k+1, k+2, \ldots
\end{array}\right.
$$

be the another solution of $L$ for $z \in T_{0} \backslash\{k \pi i: k=0,1,2,3,4\}$ with

$q^{(1)}(z)=\frac{a_{k+1}}{2 \sinh z} \operatorname{det} D, \quad q^{(2)}(z)=-\frac{a_{k+1}}{2 \sinh z} \operatorname{det} C$ 
where

$D=\left(\begin{array}{cc}f_{k+1}(-z) & \triangle f_{k+1}(-z) \\ \theta_{1} \varphi_{k-1}(z)+\theta_{2} \nabla \varphi_{k-1}(z) & \theta_{3} \varphi_{k-1}(z)+\theta_{4} \nabla \varphi_{k-1}(z)\end{array}\right)$,

then $q^{(2)}(z)=q^{(1)}(-z)=\overline{q^{(1)}(z)}$ due to $\varphi_{n}(-z)=\varphi_{n}(z)$. So, the following result can be expressed:

\section{Lemma 2.1.}

$W\left[J_{n}(z), F_{n}(z)\right]=\left\{\begin{array}{cl}p^{(2)}(z) & ; n=0,1, \ldots, k-1 \\ \operatorname{det} \theta \frac{a_{k+1}}{a_{k-2}} p^{(2)}(z) & ; n=k+1, k+2, \ldots\end{array}\right.$

for $z \in T_{0} \backslash\{k \pi i: k=0,1,2,3,4\}$.

Proof. From (6)-(9),

$$
\begin{aligned}
& W\left[J_{n}(z), F_{n}(z)\right]=a_{0}\left[J_{0}(z) F_{1}(z)-J_{1}(z) F_{0}(z)\right] \\
& =p^{(2)}(z) \\
& \text { for } n=0,1, \ldots, k-1 \text { and } \\
& W\left[J_{n}(z), F_{n}(z)\right]=a_{k+1}\left[J_{k+1}(z) F_{k+2}(z)-J_{k+2}(z) F_{k+1}(z)\right] \\
& =a_{k+1} q^{(2)}(z)\left[f_{k+1}(z) f_{k+2}(-z)-f_{k+2}(z) f_{k+1}(-z)\right] \\
& =q^{(2)}(z)(-2 \sinh z) \\
& =\operatorname{det} \theta \frac{a_{k+1}}{a_{k-2}} p^{(2)}(z)
\end{aligned}
$$

for $n=k+1, k+2, \ldots$ because $q^{(2)}(z)=-\frac{a_{k+1}}{a_{k-2}} \frac{\operatorname{det} \theta}{2 \sinh z} p^{(2)}(z)$

In addition, if $\tilde{f}_{n}(z)$ is unbounded solution of Equation (2) for $n=k+1, k+2, \ldots$ with $\lim _{n \rightarrow \infty} e^{n z} \tilde{f}_{n}(z)=$ $1, z \in \overline{\mathbb{C}}_{\text {left }}$, then

$$
W\left[f_{n}(z), \tilde{f}_{n}(z)\right]=-2 \sinh z
$$

in $z \in T \backslash\{k \pi i: k=0,1,2,3,4\}$, and the another solution of $L$

$$
G_{n}(z)=\left\{\begin{array}{cl}
\varphi_{n}(z) & ; n=0,1, \ldots, k-1 \\
r^{(1)}(z) f_{n}(z)+r^{(2)}(z) \tilde{f}_{n}(z) & ; n=k+1, k+2, \ldots
\end{array}\right.
$$

can be defined for $z \in T$ with

$r^{(1)}(z)=\frac{a_{k+1}}{2 \sinh z} \operatorname{det} E \quad, \quad r^{(2)}(z)=-\frac{a_{k+1}}{2 \sinh z} \operatorname{det} C$

where

$$
E=\left(\begin{array}{cc}
\tilde{f}_{k+1}(z) & \triangle \tilde{f}_{k+1}(z) \\
\theta_{1} \varphi_{k-1}(z)+\theta_{2} \nabla \varphi_{k-1}(z) & \theta_{3} \varphi_{k-1}(z)+\theta_{4} \nabla \varphi_{k-1}(z)
\end{array}\right)
$$

Also, the function $G_{n}(z)$ is unbounded solution of $L$ and

$$
r^{(2)}(z)=q^{(2)}(z)=\overline{q^{(1)}(z)}=-\frac{a_{k+1}}{a_{k-2}} \frac{\operatorname{det} \theta}{2 \sinh z} p^{(2)}(z)
$$


for $z \in T_{0} \backslash\{k \pi i: k=0,1,2,3,4\}$.

Theorem 2.2. For all $z$ in $T_{0} \backslash\{k \pi i: k=0,1,2,3,4\}, p^{(2)}(z) \neq 0$.

Proof. If $p^{(2)}\left(z_{0}\right)=0$ for at least $z_{0} \in T_{0} \backslash\{k \pi i: k=0,1,2,3,4\}$, then $q^{(1)}\left(z_{0}\right)=q^{(2)}\left(z_{0}\right)=0$ from (11) and hence $F_{n}\left(z_{0}\right)=0, n \in \mathbb{N} \cup\{0\}$ is trivial by using the Impulsive Conditions (4) which is a contradiction.

Definiton 2.1. The scattering function of $L$ is defined by

$S(z)=\frac{\overline{J_{0}(z)}}{J_{0}(z)}$

with respect to the Jost solution of $L$.

From the definiton of $S(z)$, it can be written that

$S(z)=\frac{\overline{p^{(2)}(z)}}{p^{(2)}(z)}=\frac{\overline{a_{0} J_{0}(z)}}{a_{0} J_{0}(z)}=\frac{J_{0}(-z)}{J_{0}(z)}=\frac{p^{(2)}(-z)}{p^{(2)}(z)}$

since $\left\{a_{n}\right\}_{n \in \mathbb{N}}$ is nonzero real sequence for all $n \in \mathbb{N} \cup\{0\}$ and $\overline{J_{n}(z)}=J_{n}(-z)$ for $z \in T_{0} \backslash$ $\{k \pi i: k=0,1,2,3,4\}$. Therefore,

$S(z)=\frac{f_{k+1}(-z)\left[\theta_{3} \varphi_{k-1}(z)+\theta_{4} \nabla \varphi_{k-1}(z)\right]-\left[\theta_{1} \varphi_{k-1}(z)+\theta_{2} \nabla \varphi_{k-1}(z)\right] \Delta f_{k+1}(-z)}{f_{k+1}(z)\left[\theta_{3} \varphi_{k-1}(z)+\theta_{4} \nabla \varphi_{k-1}(z)\right]-\left[\theta_{1} \varphi_{k-1}(z)+\theta_{2} \nabla \varphi_{k-1}(z)\right] \Delta f_{k+1}(z)}$

and so $\lim _{z \rightarrow 0} S(z)=S(0)=1$. Additionally,

$$
S(-z)=\frac{J_{0}(z)}{J_{0}(-z)}=S^{-1}(z)=\overline{S(z)}
$$

for $z \in T_{0} \backslash\{k \pi i: k=0,1,2,3,4\}$ by considering the Equalities (12).

Theorem 2.2. Let $\sigma_{d}(L)$ and $\sigma_{s s}(L)$ are the sets of eigenvalues and spectral singularities of $L$, respectively.

Then,

$\sigma_{d}(L)=\left\{\lambda \in \mathbb{C}: \lambda=2 \cosh \frac{z}{2}, z \in T_{-}, p^{(2)}(z)=0\right\}$,

$\sigma_{S S}(L)=\varnothing$

Proof. From (7), the Jost solution $J_{n}(z) \in \ell_{2}(\mathbb{N})$. Because, the first part of it consists of a finite number of elements and $f_{n}(z)$ is in $\ell_{2}(\mathbb{N})$, too. Furthermore, by using the Condition (3),

$0=J_{0}(z)=p^{(1)}(z) \varphi_{0}(z)+p^{(2)}(z) \psi_{0}(z)=\frac{p^{(2)}(z)}{a_{0}}$

and then $p^{(2)}(z)=0$. So, from the definition of spectral singularities and eigenvalues in [27] and Theorem 2.2.,

$\sigma_{d}(L)=\left\{\lambda \in \mathbb{C}: \lambda=2 \cosh \frac{z}{2}, z \in T_{-}, p^{(2)}(z)=0\right\}$

and

$\sigma_{s S}(L)=\left\{\lambda \in \mathbb{C}: \lambda=2 \cosh \frac{z}{2}, z \in T_{0} \backslash\{k \pi i: k=0,1,2,3,4\}, p^{(2)}(z)=0\right\}$ 


$$
=\emptyset \text {. }
$$

Note that, the Wronskian of $J_{n}(z)$ and $G_{n}(z)$ can be written from (7) and (10) as

$W\left[J_{n}(z), G_{n}(z)\right]=\left\{\begin{array}{cl}p^{(2)}(z) & ; n=0,1, \ldots, k-1 \\ \operatorname{det} \theta \frac{a_{k+1}}{a_{k-2}} p^{(2)}(z) & ; n=k+1, k+2, \ldots\end{array}\right.$

for $z \in T$. For this reason, since the last theorem is taken into account, the quantitative properties of zeros of $p^{(2)}(z)$ in $T_{-}$are required to investigate the quantitative properties of the eigenvalues of $L$.

\section{AN EXAMPLE}

Let $L_{1}$ denote the operator in $\ell_{2}(\mathbb{N})$ generated by the equation

$y_{n-1}+y_{n+1}=\left(2-\lambda^{2}\right) y_{n}, \quad n \in \mathbb{N} \backslash\{3,4,5\}$,

with boundary and impulsive conditions

$$
\begin{gathered}
y_{0}=0 \\
\left(\begin{array}{c}
y_{5} \\
\Delta y_{5}
\end{array}\right)=\theta\left(\begin{array}{c}
y_{3} \\
\nabla y_{3}
\end{array}\right)
\end{gathered}
$$

where $\operatorname{det} \theta \neq 0$ for $\theta=\left(\begin{array}{ll}\theta_{1} & \theta_{2} \\ \theta_{3} & \theta_{4}\end{array}\right),\left\{\theta_{i}\right\}_{i=1,2,3,4} \in \mathbb{R}$ and $\lambda=2 \cosh \left(\frac{z}{2}\right)$ is a hyperbolic eigenparameter.

Moreover, $\left\{\varphi_{n}(z)\right\}$ and $\left\{\psi_{n}(z)\right\}, n=0,1,2,3$ are the elementary solutions of (14) for $z \in T$ subject to the Initial Conditions (6) and clearly $f_{n}(z)=\alpha_{n} e^{n z}$ where $\alpha_{n+1}=-\alpha_{n}$. Also, it can be written from (14) and (15)

$$
\begin{array}{ll}
\varphi_{2}(\lambda)=2-\lambda^{2}, & \varphi_{3}(\lambda)=\lambda^{4}-4 \lambda^{2}+3 \\
\psi_{2}(\lambda)=-1, & \psi_{3}(\lambda)=\lambda^{2}-2
\end{array}
$$

and

$$
\begin{aligned}
p^{(1)}(z)= & \frac{1}{\operatorname{det} \theta}\left\{\left[\theta_{1} \psi_{3}(z)+\theta_{2} \nabla \psi_{3}(z)\right] \triangle f_{5}(z)-f_{5}(z)\left[\theta_{3} \psi_{3}(z)+\theta_{4} \nabla \psi_{3}(z)\right]\right\} \\
= & -\frac{\alpha_{5}}{\operatorname{det} \theta} e^{4 z}\left[\left(\theta_{1}+\theta_{2}\right) e^{3 z}+\left(\theta_{1}+2 \theta_{2}+\theta_{3}+\theta_{4}\right) e^{2 z}+\left(\theta_{1}+2 \theta_{2}+\theta_{4}\right) e^{z}\right. \\
& \left.+\left(\theta_{1}+\theta_{2}+\theta_{3}+\theta_{4}\right)\right], \\
p^{(2)}(z)= & -\frac{1}{\operatorname{det} \theta}\left\{\left[\theta_{1} \varphi_{3}(z)+\theta_{2} \nabla \varphi_{3}(z)\right] \triangle f_{5}(z)-f_{5}(z)\left[\theta_{3} \varphi_{3}(z)+\theta_{4} \nabla \varphi_{3}(z)\right]\right\} \\
= & \frac{\alpha_{5}}{\operatorname{det} \theta} e^{3 z}\left[\left(\theta_{1}+\theta_{2}\right) e^{5 z}+\left(\theta_{1}+\theta_{3}+\theta_{4}\right) e^{4 z}+\left(\theta_{1}-\theta_{4}\right) e^{3 z}+\left(\theta_{1}+\theta_{3}+\theta_{4}\right) e^{2 z}\right. \\
& \left.+\left(\theta_{1}-\theta_{4}\right) e^{z}+\left(\theta_{1}+\theta_{2}+\theta_{3}+\theta_{4}\right)\right] .
\end{aligned}
$$

By following the Equality (7),

$J_{n}(z)=\left\{\begin{array}{cl}\alpha_{5} e^{5 z}\left[p^{(11)}(z) \varphi_{n}(z)+p^{(21)}(z) \psi_{n}(z)\right] & ; n=0,1,2,3 \\ -\alpha_{n} e^{n z} & ; n=5,6,7, \ldots\end{array}\right.$

is the Jost solution of $L_{1}$ where

$$
\begin{aligned}
& p^{(11)}(z)=-\frac{\left[\theta_{1}\left(\lambda^{2}-2\right)+\theta_{2}\left(\lambda^{2}-1\right)\right]\left(e^{z}+1\right)+\theta_{3}\left(\lambda^{2}-2\right)+\theta_{4}\left(\lambda^{2}-1\right)}{\operatorname{det} \theta}, \\
& p^{(21)}(z)=\frac{\left[\theta_{1}\left(\lambda^{4}-4 \lambda^{2}+3\right)+\theta_{2}\left(\lambda^{4}-3 \lambda^{2}+1\right)\right]\left(e^{z}+1\right)+\theta_{3}\left(\lambda^{4}-4 \lambda^{2}+3\right)+\theta_{4}\left(\lambda^{4}-3 \lambda^{2}+1\right)}{\operatorname{det} \theta} .
\end{aligned}
$$


From (13), the scattering function of $L_{1}$ is

$$
\begin{aligned}
S(z) & =\frac{\left[\theta_{1} \varphi_{3}(z)+\theta_{2} \nabla \varphi_{3}(z)\right] \Delta f_{5}(-z)-f_{5}(-z)\left[\theta_{3} \varphi_{3}(z)+\theta_{4} \nabla \varphi_{3}(z)\right]}{\left[\theta_{1} \varphi_{3}(z)+\theta_{2} \nabla \varphi_{3}(z)\right] \Delta f_{5}(z)-f_{5}(z)\left[\theta_{3} \varphi_{3}(z)+\theta_{4} \nabla \varphi_{3}(z)\right]} \\
& =e^{-6 z\left[\frac{t(-z)}{t(z)}\right]}
\end{aligned}
$$

for $z \in T_{0} \backslash\{k \pi i: k=0,1,2,3,4\}$ where

$$
\begin{aligned}
t(z)= & {\left[\left(\theta_{1}+\theta_{2}\right) e^{5 z}+\left(\theta_{1}+\theta_{3}+\theta_{4}\right) e^{4 z}+\left(\theta_{1}-\theta_{4}\right) e^{3 z}+\left(\theta_{1}+\theta_{3}+\theta_{4}\right) e^{2 z}+\left(\theta_{1}-\theta_{4}\right) e^{z}\right.} \\
& \left.+\left(\theta_{1}+\theta_{2}+\theta_{3}+\theta_{4}\right)\right] .
\end{aligned}
$$

In addition, the eigenvalues of $L_{1}$ is

$$
\sigma_{d}\left(L_{1}\right)=\left\{\lambda \in \mathbb{C}: \lambda=2 \cosh \frac{z}{2}, z \in T_{-}, p^{(2)}(z)=0\right\},
$$

so

$t(z)=0$

can be obtained because $\lambda=2 \cosh \frac{z}{2}$ and $p^{(2)}(z)=0$ in $\sigma_{d}\left(L_{1}\right)$.

Case 1: If $\theta=I_{2}$ where $I_{2}$ is $2 \times 2$ identity matrix, then

$e^{5 z}+2 e^{4 z}+2 e^{2 z}+2=0$

from using (16). After that,

$$
\begin{aligned}
& e^{z} \approx-2.40541 \\
& e^{z} \approx-0.370293-i 0.872969 \\
& e^{z} \approx-0.370293+i 0.872969 \\
& e^{z} \approx 0.572995-i 0.772239 \\
& e^{z} \approx 0.572995+i 0.772239
\end{aligned}
$$

and $z_{1} \approx-0.05312+i 4.31122, z_{2} \approx-0.05312+i 10.59441, z_{3} \approx-0.05312+i 8.25516$,

$z_{4} \approx-0.03915+i 4.07404, z_{5} \approx-0.03915+i 10.35723, z_{6} \approx-0.03915+i 8.49234$ are the roots of (17) in $T_{-}$, then the eigenvalues of $L_{1}$ is $\sigma_{d}\left(L_{1}\right)=\left\{\lambda \in \mathbb{C}: \lambda=2 \cosh \frac{z_{k}}{2} ; k=1,2,3,4,5,6\right\}$.

Case 2: For $\theta=\left[\begin{array}{ll}0 & 1 \\ 1 & 0\end{array}\right]$, it can be found that

$e^{5 z}+e^{4 z}+e^{2 z}+2=0$.

From the last equation,

$$
\begin{aligned}
& e^{z} \approx-1.6439 \\
& e^{z} \approx-0.398336-i 0.97864 \\
& e^{z} \approx-0.398336+i 0.97864 \\
& e^{z} \approx 0.720286-i 0.755613 \\
& e^{z} \approx 0.720286+i 0.755613
\end{aligned}
$$

Since there is no root of (18), the eigenvalues of $L_{1}$ is empty. 


\section{CONFLICTS OF INTEREST}

No conflict of interest was declared by the author.

\section{REFERENCES}

[1] Agarwal, R. P., "Difference equation and inequalities: Theory, methods and applications", Marcel Dekkar Inc., New York, Basel, (2000).

[2] Aygar, Y., "The effects of hyperbolic eigenparameter on spectral analysis of a quantum difference equations", Malaysian Journal of Mathematical Sciences, 11(3): 317-330, (2017).

[3] Bairamov, E., Aygar, Y., Koprubasi, T., "The spectrum of eigenparameter-dependent discrete Sturm-Liouville equations", Journal of Computational and Applied Mathematics, 235(16): 45194523, (2011).

[4] Dolzhenko, E. P., "Boundary value uniqueness theorems for analytic functions", Mathematical Notes, 25: 437-442, (1979).

[5] Guseinov, G. S., "The determination of an infinite Jacobi Matrix from the scattering date", Soviet Mathematics Doklady, 17: 596-600, (1976).

[6] Hislop, P. D., Sigal, I. M., "Introduction to spectral theory with applications to Schrödinger operators", Springer, New York, (1996).

[7] Kelley, W.G., Peterson, A.C., "Difference equations: An introduction with applications", Harcourt Academic Press, San Diego, (2001).

[8] Koprubasi, T., Mohapatra, R. N., "Spectral properties of generalized eigenparameter dependent discrete Sturm-Liouville type equation”, Quaestiones Mathematicae, 40(4): 491-505, (2017).

[9] Naimark, M. A., "Linear differential operators II”, Ungar, New York, (1968).

[10] Yokus, N., Coskun, N., "Jost solution and the spectrum of the discrete Sturm-Liouville equations with hyperbolic eigenparameter", Neural, Parallel and Scientific Computations, 24: 419-430, (2016).

[11] Bairamov, E., "Spectral properties of the nonhomogeneous Klein-Gordon s-wave equations", Rocky Mountain Journal of Mathematics, 34(1): 1-11, (2004).

[12] Bairamov, E., Cakar, O., Celebi, A. O., "Quadratic pencil of Schrödinger operators with spectral singularities: Discrete spectrum and principal functions", Journal of Mathematical Analysis and Applications, 216(1): 303-320, (1997).

[13] Bairamov, E., Cakar, O., Krall, A.M., "An eigenfunction expansion for a quadratic pencil of a Schrödinger operator with spectral singularities", Journal of Differential Equations, 151(2): 268289, (1999).

[14] Bairamov, E., Cebesoy, S., Erdal, I.,"Properties of eigenvalues and spectral singularities for impulsive quadratic pencil of difference operators", Journal of Applied Analysis \& Computation, 9(4): 1454-1469, (2019).

[15] Bairamov, E., Celebi, A. O., "Spectral properties of the Klein-Gordon s-wave equation with complex potential", Indian Journal of Pure and Applied Mathematics, 28(6): 813-824, (1997). 
[16] Bairamov, E., Karaman, O., "Spectral singularities of Klein-Gordon s-wave equation with an integral boundary condition", Acta Mathematica Hungarica, 97: 121-131, (2002).

[17] Coskun, N., Yokus, N., "A Note on the spectrum of discrete Klein-Gordon s-wave equation with eigenparameter dependent boundary condition", Filomat, 33(2): 449-455, (2019).

[18] Degasperis, A., “On the inverse problem for the Klein-Gordon s-wave equation”, Journal of Mathematical Physics, 11(2): 551-567, (1970).

[19] Krall, A.M., Bairamov, E., Cakar, O.,"Spectrum and spectral singularities of a quadratic pencil of a Schrödinger operator with a general boundary condition”, Journal of Differential Equations, 151(2): 252-267, (1999).

[20] Allahverdiev, B.P., Bairamov, E., Ugurlu, E., "Eigenparameter dependent Sturm-Liouville problems in boundary conditions with transmission conditions", Journal of Mathematical Analysis and Applications, 401(1): 388-396, (2013).

[21] Bairamov, E., Aygar, Y., Karslioglu, D., "Scattering analysis and spectrum of discrete Schrödinger equations with transmission conditions", Filomat, 31(17): 5391-5399, (2017).

[22] Lakshmikantham, V., Bainov, D. D., Simeonov, P. S., "Theory of Impulsive Differential Equations", World Scientific Publishing Co. Inc., Teaneck, NJ, (1989).

[23] Mukhtarov, Sh. O., Tunc, E., "Eigenvalue problems for Sturm-Liouville equations with transmission conditions", Israel Journal of Mathematics, 144: 367-380, (2004).

[24] Ugurlu, E., Bairamov, E., "Dissipative operators with impulsive conditions", Journal of Mathematical Chemistry, 51: 1670-1680, (2013).

[25] Ugurlu, E., Bairamov, E., "Krein's theorems for a dissipative boundary value transmission problem", Complex Analysis and Operator Theory, 7: 831-842, (2013).

[26] Adivar, M., "Quadratic pencil of difference equations: Jost solutions, spectrum, and principal vectors", Quaestiones Mathematicae, 33(3): 305-323, (2010).

[27] Berezanski, Y. M., "Expansions in eigenfunctions of selfadjoint operators", AMS, Providence, (1968). 